\title{
Thermal Therapy in Patients Suffering from Non-Specific Chronic Low Back Pain-A Systematic Review
}

\author{
Selina Wittenwiler ${ }^{1}$, Rahel Stoop ${ }^{1}$, Erich Hohenauer ${ }^{1,2,3}$, Ron Clijsen ${ }^{1,2,3}$ \\ ${ }^{1}$ Research Rehabilitation 2rLab, Department of Business Economics, Health and Social Care, University of Applied Sciences and \\ Arts of Southern Switzerland, Landquart, Switzerland \\ ${ }^{2}$ THIM University of Applied Sciences, Landquart, Switzerland \\ ${ }^{3}$ Department of Movement and Sport Sciences, Vrije Universiteit Brussel, Brussels, Belgium \\ Email: rahel.stoop@supsi.ch
}

How to cite this paper: Wittenwiler, $\mathrm{S}$. Stoop, R., Hohenauer, E. and Clijsen, R. (2018) Thermal Therapy in Patients Suffering from Non-Specific Chronic Low Back Pain-A Systematic Review. International Journal of Clinical Medicine, 9, 294-314.

https://doi.org/10.4236/ijcm.2018.94026

Received: March 17, 2018

Accepted: April 25, 2018

Published: April 28, 2018

Copyright (c) 2018 by authors and Scientific Research Publishing Inc. This work is licensed under the Creative Commons Attribution International License (CC BY 4.0).

http://creativecommons.org/licenses/by/4.0/

\begin{abstract}
Thermal therapy is frequently used as an adjunct to treatment in patients suffering from chronic low back pain. It is also an inherent part of patients' self-administered pain treatment. This review aims to update the evidence for thermal therapy treatments in non-specific chronic low back pain patients and to rate the methodological quality of the corresponding clinical trials. Previous studies have reported contradictory evidence for the effectiveness of thermal therapy. An electronic search on MEDLINE (PubMed), PEDro, CENTRAL and CINHAL databases was conducted between May 2016 and February 2018. Clinical trials comparing local thermal therapy to conservative or no treatment were assessed for eligibility. Pain, physical function and global health were defined as outcome parameters. A total of $\mathrm{n}=9$ studies met the inclusion criteria. All of them applied an electrophysical agent as the thermal treatment: continuous ultrasound $(n=6)$, short-wave diathermy $(n=$ $2)$, microwave diathermy $(n=1)$. Out of the $n=6$ studies on ultrasound treatment, $\mathrm{n}=2$ reported significant within and between-group results for pain reduction after 4 to 6 weeks of treatment. Both short-wave diathermy studies demonstrated significant between-group results for pain reduction after 3 weeks of treatment. Contradictory results for all other observed outcome parameters were reported regardless of the intervention. Moreover, significant within-group results for the control groups questioned the effectiveness of the intervention treatments. Therefore, the effect of thermal therapy, (electrophysical agents), is not superior to any control treatment except for ultrasound treatment on short-term pain reduction.
\end{abstract}




\section{Keywords}

Electrophysical Agents, Heat Application, Pain Reduction, Physical Function, Non-Specific Chronic Low Back Pain, Thermal Therapy

\section{Introduction}

According to the World Health Organization, chronic pain is a worldwide public health problem [1]. It is defined as "pain lasting longer than normal tissue healing time", generally taken to be 12 weeks [2]. In looking at the prevalence of chronic low back pain in US citizens over a period of 14 years, there was a significant increase of about $6.3 \%$ with no changes in symptom severity or general health [3]. Chronic pain impairs quality of life, work performance and increases healthcare costs [4]. The socio-economic burden of low back pain has been evaluated by several studies [5] [6] [7]. Approximately $80 \%$ of low back pain cases are non-specific, meaning that no definite anatomical structure can be associated with neither the medical history nor the clinical examination [8]. Despite existing recommendations for avoiding chronicity of low back pain [9], non-specific chronic low back pain (NSCLBP) continues to be increasing in prevalence in both developed and developing countries [10]. Several countries provide corresponding treatment and behaviour guidelines [11] highlighting the benefits of physical activity [12]. Strong evidence exists to encourage chronic low back pain patients to follow an active lifestyle and to assume self-responsibility for their health [13]. Despite conflicting evidence [14] [15] [16] [17], thermal therapy is applied in practice and by patients themselves as a self-management strategy [18]. Thermal therapy comprises the implementation of any superficial heat or warmth application to the skin via conduction or convection [19]. Thermal therapy is believed to influence pain [20], to increase superficial and deep muscle tissue temperature and blood flow [21] affecting muscle nerve conduction velocity [22]. Electrophysical agents generate heat within the tissue by means of energy conversion (e.g. ultrasound, diathermy) [19]. Allen (2009) stated that "physical agents may serve as useful adjunctive modalities of pain relief or to enhance the effectiveness of other elements in therapy geared toward resolution of movement impairments and restoration of physical function" [15]. Despite these findings, the European guidelines for the management of NSCLBP (2006) do not consider thermal therapy to be more effective than placebo or other treatments neither to relieve pain, nor to improve functional outcome parameters [17].

Thus, the authors of this review wanted to update the evidence for thermal therapy applications in patients with NSCLBP with respect to pain reduction and improvement of functional outcome parameters and global health and to rate the methodological quality of the included studies. 


\section{Methods}

\subsection{Research Question}

The research question was defined by the PICOS-model [23] in accordance with the Preferred Reporting Items for Systematic Reviews and Meta-Analyses (PRISMA) statement [24]: Population: patients diagnosed with NSCLBP (>3 months symtomatic) aged 18 years or older; Intervention: thermal therapy applications except balneo/spa therapy; Comparator: any kind of treatment except thermal therapy; Outcomes: pain ratings, physical function, global health; Study design: RCT, CCT, CT.

\subsection{Literature Search Strategies and Data Sources}

An electronic systematic search, according to the guidelines of the PRISMA statement [24], was conducted between May 2016 and February 2018 on the MEDLINE (PubMed), PEDro (Physiotherapy Evidence Database), CENTRAL (Cochrane Central Register of Controlled Trials), and CINHAL (Cumulative Index to Nursing and Allied Health Literature) databases. The additional filters "randomized controlled trials, clinical trials, controlled clinical trials, English language, full text" were applied in PubMed, the filter "clinical trials" in PEDro, "trials" in CENTRAL and "full text, academic journal" in CINHAL. Grey literature was searched on the websites of Google Scholar, Aspetar Sports Medicine Journal and by screening the Book of Abstracts of the European College of Sports Science (2014-2017). The function "similar articles" on PubMed and screening the reference lists were applied to attain additional literature. MeSH-proven (Medical Subject Headings) keywords were applied where possible (eAddenda Appendix I). The keywords which best fitted the research question among others were: "low back pain", "heat application", "hot packs", "thermal therapy", "warmth application". The keywords representing the thermal therapy application were always combined with "AND" and "low back pain" (Table 1). The a priori set inclusion criteria were: 1) RCT, CCT, CT, 2) English full-text availability, 3) participants of 18 years or older diagnosed with NSCLBP, 4) control intervention of any treatment including placebo/sham without using thermal therapy, 5) outcome parameters comprising pain ratings and/or physical function and/or global health. The rationale for inclusion of these outcome variables was comparability. After screening and processing all the articles found $(\mathrm{n}=164)$, a total of $\mathrm{n}=9$ articles was included in the final data analysis. Figure 1 shows the flow-chart of the selection process.

\subsection{Data Extraction and Quality Assessment}

The methodological quality of the studies was assessed using the 11-item PEDro scale [25] [26] and the Cochrane Risk of Bias Tool (ROB) [27]. Two researchers (SW, RS) rated independently from each other the $n=9$ studies. In case of disagreement or doubt consensus was reached by a third investigator (EH). For trials which PEDro scores were originally listed on the PEDro website 
Table 1. Summary of keywords and its combinations of each database.

\begin{tabular}{|c|c|c|c|c|c|c|}
\hline \multirow{2}{*}{$\frac{\text { 1) Keyword }}{\text { low back pain }}$} & \multirow{2}{*}{$\frac{\text { Boolean function }}{\text { AND }}$} & \multirow{2}{*}{$\begin{array}{l}\text { 2) Keyword } \\
\text { diathermy }\end{array}$} & \multicolumn{4}{|c|}{ Hits per Database } \\
\hline & & & PubMed: $\mathrm{n}=10$ & PEDro: $\mathrm{n}=12$ & CENTRAL: $\mathrm{n}=23$ & CINHAL: $\mathrm{n}=14$ \\
\hline & & fango packs & PubMed: $\mathrm{n}=0$ & PEDro: $\mathrm{n}=0$ & CENTRAL: $\mathrm{n}=0$ & CINHAL: $\mathrm{n}=0$ \\
\hline & & heat application & PubMed: $\mathrm{n}=0$ & PEDro: $\mathrm{n}=1$ & CENTRAL: $\mathrm{n}=1$ & CINHAL: $\mathrm{n}=32$ \\
\hline & & heat plaster & PubMed: $\mathrm{n}=1$ & PEDro: $\mathrm{n}=0$ & CENTRAL: $\mathrm{n}=0$ & CINHAL: $\mathrm{n}=1$ \\
\hline & & heat wrap & PubMed: $\mathrm{n}=4$ & PEDro: $\mathrm{n}=5$ & CENTRAL: $\mathrm{n}=8$ & CINHAL: $\mathrm{n}=16$ \\
\hline & & hot packs & PubMed: $\mathrm{n}=4$ & PEDro: $\mathrm{n}=5$ & CENTRAL: $\mathrm{n}=21$ & CINHAL: $\mathrm{n}=16$ \\
\hline & & hyperemic cream & PubMed: $\mathrm{n}=0$ & PEDro: $\mathrm{n}=0$ & CENTRAL: $\mathrm{n}=0$ & CINHAL: $\mathrm{n}=0$ \\
\hline & & hyperemic gel & PubMed: $\mathrm{n}=0$ & PEDro: $\mathrm{n}=0$ & CENTRAL: $\mathrm{n}=0$ & CINHAL: $\mathrm{n}=0$ \\
\hline & & hyperemic ointment & PubMed: $\mathrm{n}=0$ & PEDro: $\mathrm{n}=0$ & CENTRAL: $\mathrm{n}=0$ & CINHAL: $\mathrm{n}=0$ \\
\hline & & hyperemic products & PubMed: $\mathrm{n}=0$ & PEDro: $\mathrm{n}=0$ & CENTRAL: $\mathrm{n}=0$ & CINHAL: $\mathrm{n}=0$ \\
\hline & & hyperemia induced & PubMed: $\mathrm{n}=13$ & PEDro: $\mathrm{n}=0$ & CENTRAL: $\mathrm{n}=12$ & CINHAL: $\mathrm{n}=9$ \\
\hline & & mud packs & PubMed: $\mathrm{n}=1$ & PEDro: $\mathrm{n}=0$ & CENTRAL: $\mathrm{n}=1$ & CINHAL: $\mathrm{n}=1$ \\
\hline & & thermal therapy & PubMed: $\mathrm{n}=5$ & PEDro: $\mathrm{n}=8$ & CENTRAL: $\mathrm{n}=6$ & CINHAL: $\mathrm{n}=15$ \\
\hline & & thermotherapy & PubMed: $\mathrm{n}=4$ & PEDro: $\mathrm{n}=3$ & CENTRAL: $\mathrm{n}=13$ & CINHAL: $\mathrm{n}=4$ \\
\hline & & $\begin{array}{l}\text { topical hyperemic } \\
\text { products }\end{array}$ & PubMed: $\mathrm{n}=0$ & PEDro: $\mathrm{n}=0$ & CENTRAL: $\mathrm{n}=0$ & CINHAL: $\mathrm{n}=0$ \\
\hline & & $\begin{array}{c}\text { topical warming } \\
\text { product }\end{array}$ & PubMed: $\mathrm{n}=0$ & PEDro: $\mathrm{n}=0$ & CENTRAL: $\mathrm{n}=0$ & CINHAL: $\mathrm{n}=0$ \\
\hline & & ultrasonic therapy & PubMed: $\mathrm{n}=9$ & PEDro: $\mathrm{n}=30$ & CENTRAL: $\mathrm{n}=27$ & CINHAL: $\mathrm{n}=24$ \\
\hline & & warming products & PubMed: $\mathrm{n}=0$ & PEDro: $\mathrm{n}=0$ & CENTRAL: $\mathrm{n}=0$ & CINHAL: $\mathrm{n}=0$ \\
\hline & & warming application & PubMed: $\mathrm{n}=0$ & PEDro: $\mathrm{n}=0$ & CENTRAL: $\mathrm{n}=0$ & CINHAL: $\mathrm{n}=0$ \\
\hline
\end{tabular}

(https://www.pedro.org.au/) [26], the presented PEDro score was used. The data extraction was performed in the same manner. Data on study design, participants, intervention method, assessment and each outcome parameter were split into corresponding columns on spreadsheets to extract relevant data. The accepted level of significance was set at alpha $<0.05$. The between-group difference results were extracted following the completion of the whole treatment in order to observe the overall effect of the specified thermal therapy versus the control intervention.

\section{Results}

\subsection{Total PEDro Score and Risk of Bias Analysis}

The total PEDro scores (eAddenda Appendix II) of the included studies were in line with the Risk of Bias results. Figure 2 depicts the Risk of Bias results of each included study and Figure 3 demonstrates the overview of all included studies for this analysis. A low risk of reporting bias was observed in $100 \%$ of the analysed studies. A high risk of bias with $>50 \%$ contributed to performance and detection bias (Personnel and Outcome assessor) as well as attrition bias. A low risk of bias with $>75 \%$ was attained for selection and other bias. 

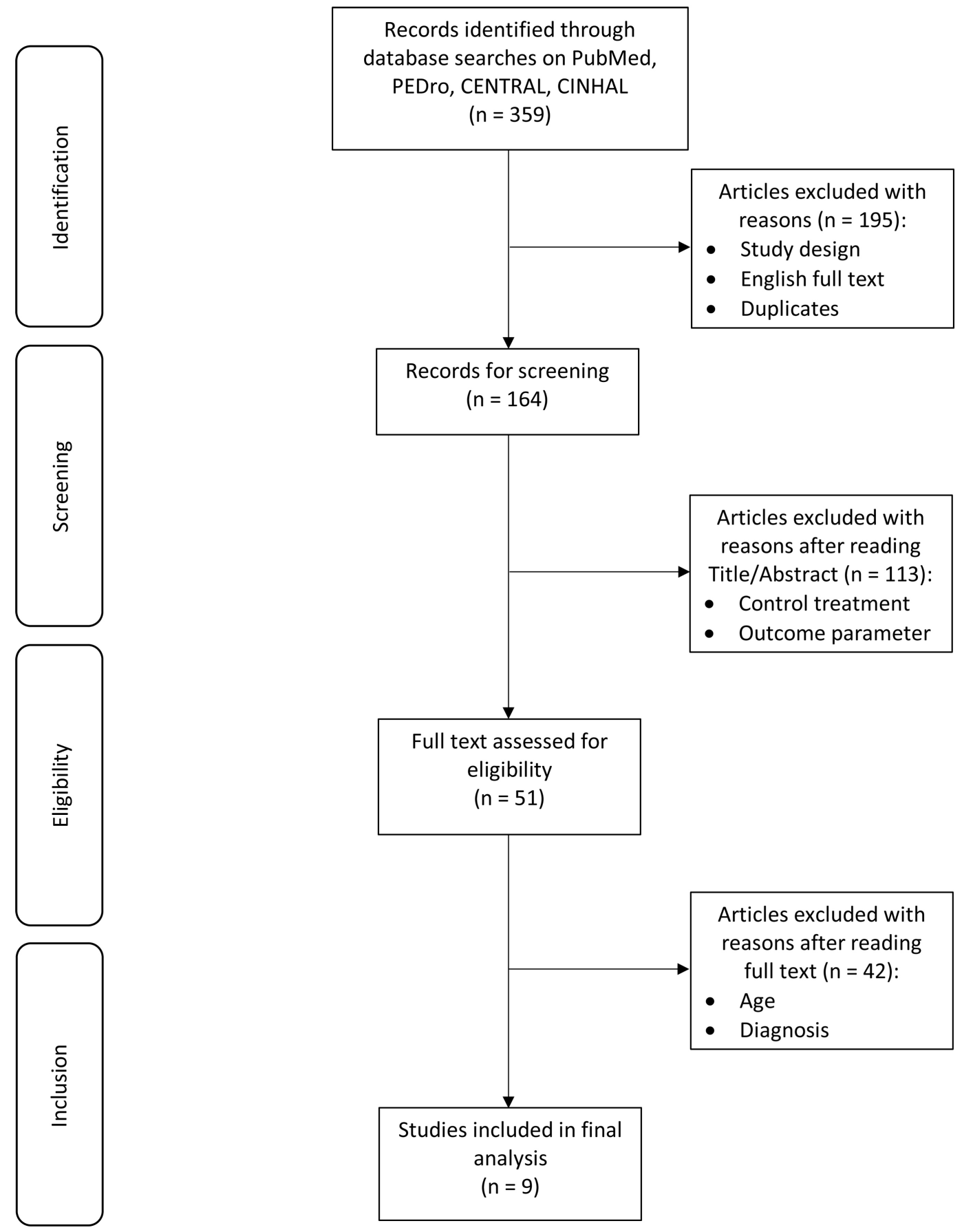

Figure 1. Flow-chart describing the selection process.

\subsection{Study Characteristics}

All included studies exclusively applied electrophysical agents as intervention treatment; $\mathrm{n}=6$ studies used ultrasound treatment [28]-[33], $\mathrm{n}=2$ studies applied short-wave diathermy [34] [35] and $\mathrm{n}=1$ study microwave diathermy [36] (see Figure 4 for detailed overview on the results). All studies, except Licciardone et al. (2013) [32] (USA), were performed in Eastern countries ( $\mathrm{n}=3$ Iran [28] [31] [33], $\mathrm{n}=3$ Turkey [29] [30] [36], $\mathrm{n}=2$ Bangladesh [34] [35]. The gender 


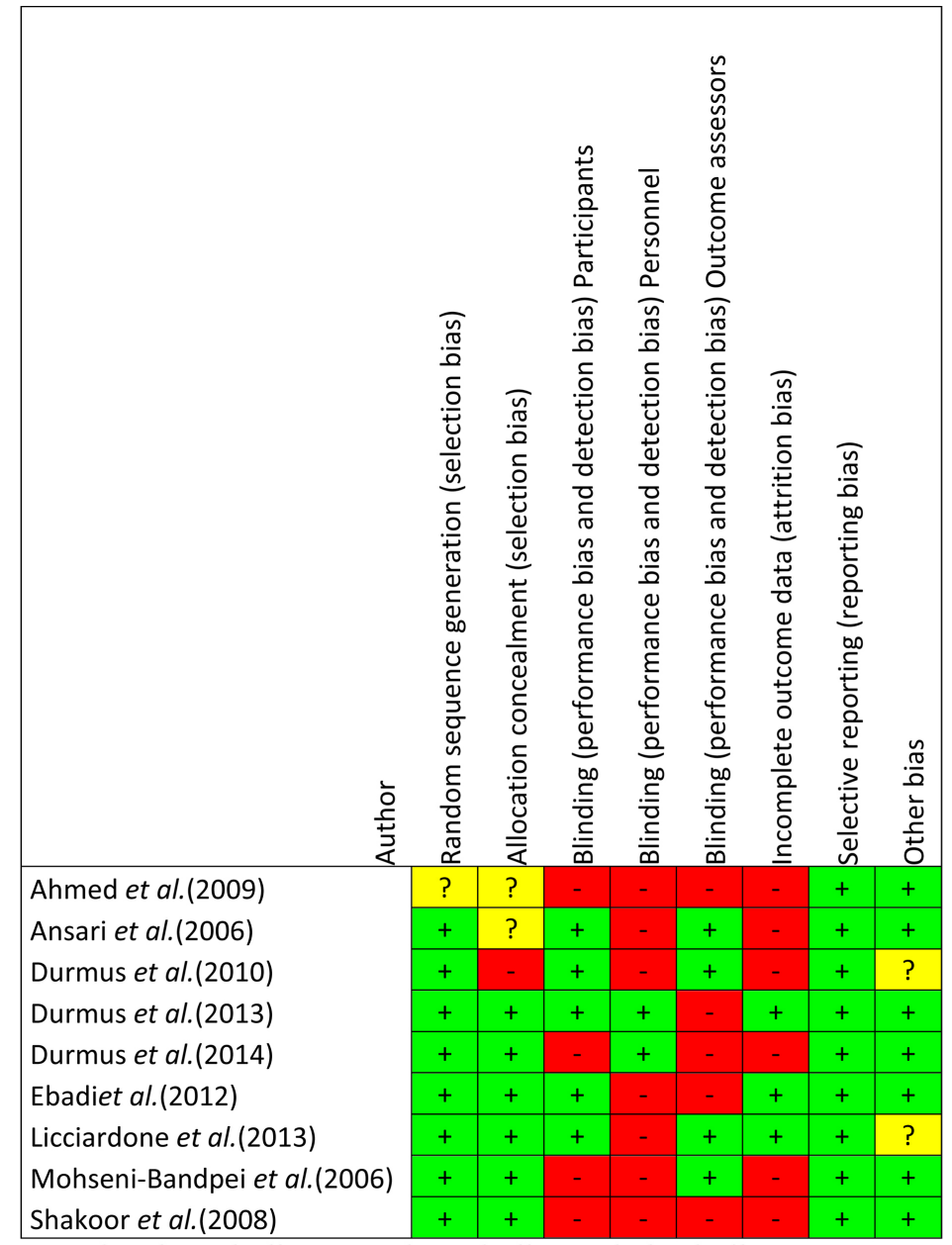

Legend: + = low risk of bias, ? = unclear risk of bias, - = high risk of bias.

Figure 2. Risk of bias graph for each included study.

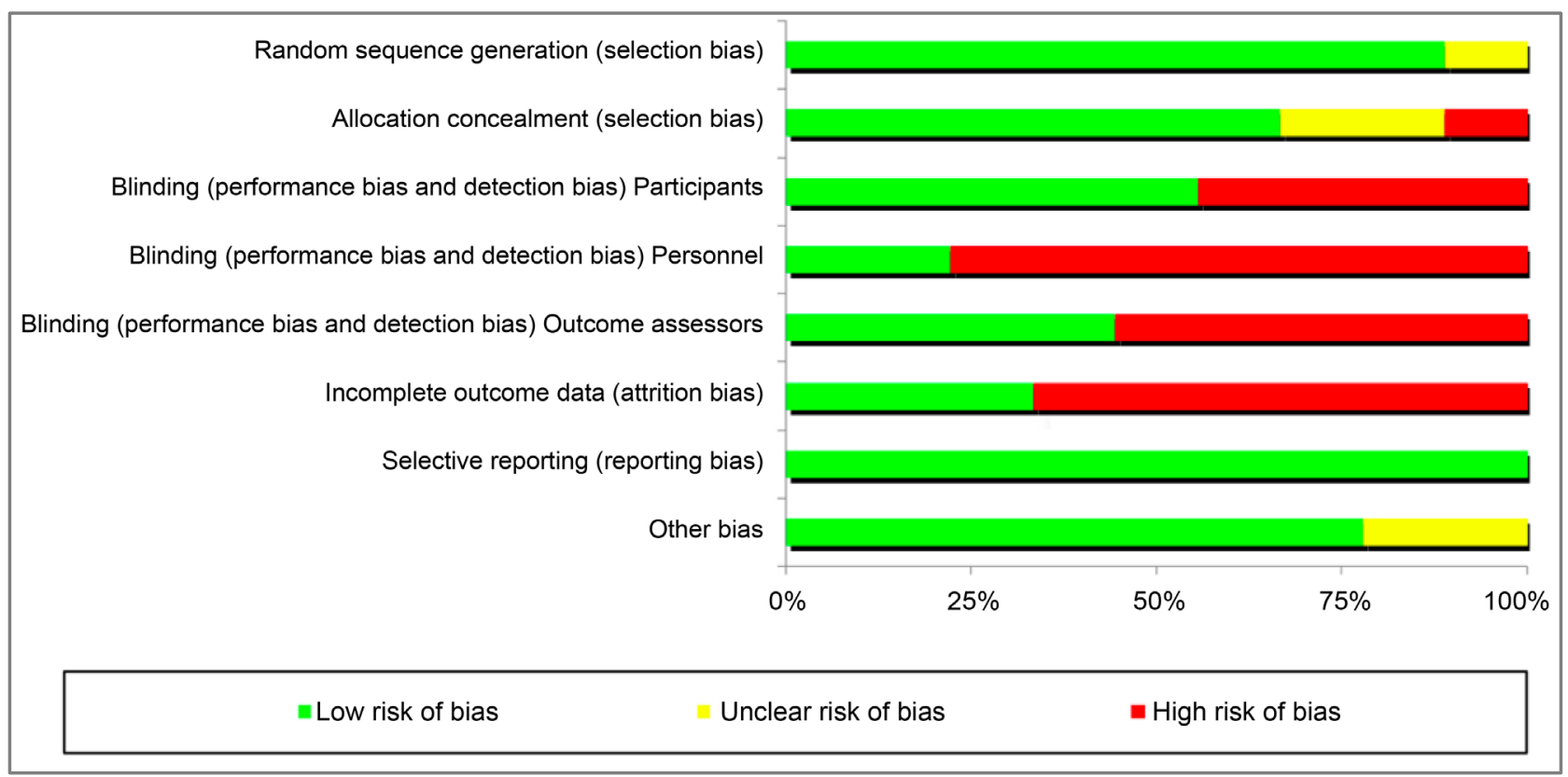

Figure 3. Risk of bias summary for all included studies. 


\begin{tabular}{|c|c|c|c|c|c|c|c|}
\hline $\begin{array}{l}\text { Authors } \\
\text { Study } \\
\text { Design } \\
\text { Meth. } \\
\text { Quality }\end{array}$ & $\begin{array}{c}\text { Population } \\
\text { characteristics } \\
\text { mean } \pm \text { SD }\end{array}$ & Interventions & Outcomes & $\begin{array}{c}\text { Measurement time } \\
\text { points } \\
\text { Follow-up }\end{array}$ & & $\begin{array}{l}\text { Results } \\
\text { p }<0.05 \S\end{array}$ & \\
\hline \multirow{3}{*}{$\begin{array}{l}\text { Ahmed et } \\
\text { al. (2009) }\end{array}$} & \multirow{2}{*}{$\begin{array}{l}\text { Bangladesh } \\
\mathrm{N}=111\end{array}$} & \multirow{2}{*}{ IG: SWD } & \multirow{3}{*}{$\begin{array}{l}\text { Lattinnen's test } \\
\text { score }(0-20 \\
\text { points): }\end{array}$} & Baseline (M0) & \multicolumn{3}{|c|}{ Sum of Lattinnen's test score, Tenderness score and VAS ( $0-34$ points $)$} \\
\hline & & & & End of 1 th wk (M1) & SWD $(I G, N=47)$ & pSWD (CG, N = 50) & Sign. IG vs CG \\
\hline & Male: $\mathrm{N}=65$ & & & End of $2 \mathrm{nd} w \mathrm{k}(\mathrm{M} 2)$ & M0: $20.44 \pm 3.02$ & M0: $20.10 \pm 3.51$ & M0: -0.97 to 1.66 \\
\hline RCT & Female: $\mathrm{N}=46$ & Time: $6 \mathrm{wk}$ & Pain intensity & End of 3th wk (M3) & M1: $17.82 \pm 3.79$ & M1: $18.78 \pm 3.21$ & $p=0.63$ \\
\hline & DO: $N=14$ & & Pain frequency & End of 4 th wk (M4) & $M 2: 15.34 \pm 4.82$ & M2: $17.58 \pm 3.39$ & M1: -2.37 to 0.47 \\
\hline \multirow{10}{*}{$\begin{array}{l}\text { PEDro- } \\
\text { score: } \\
4 / 10\end{array}$} & & IG, CG identical EP, & Analgesic intake & End of 5 th wk (M5) & M3: $13.06 \pm 5.01$ & $M 3: 15.70 \pm 3.77$ & $p=0.14$ \\
\hline & IG: $N=47$ & information, medi- & Disability & End of 6 th wk (M6) & $M 4: 11.06 \pm 4.15$ & $M 4: 15.04 \pm 3.77$ & M2: 3.93 to -0.54 \\
\hline & CG: $N=50$ & cation & Sleep disturbance & & M5: $8.34 \pm 3.62$ & M5: $14.02 \pm 3.31$ & $\mathrm{p}=0.01^{\S}$ \\
\hline & $\begin{array}{l}\text { Age (y): } \\
39 \pm 11.94\end{array}$ & $\begin{array}{l}\text { SWD: } 3 \mathrm{x} / \text { wk for } 15 \\
\text { min on lumbar } \\
\text { spine }\end{array}$ & $\begin{array}{l}\text { Tenderness score } \\
(0-4 \text { points })\end{array}$ & No FU & M6: $6.44 \pm 3.06$ & M6: $13.39 \pm 3.10$ & $\begin{array}{l}\text { M3: } 4.43 \text { to }-0.83 \\
p=0.005 \S \\
\text { M4: }-5.58 \text { to }-2.37 \\
p=0.0 \S\end{array}$ \\
\hline & $\mathrm{BMI}^{\#}\left(\mathrm{~kg} / \mathrm{m}^{2}\right):$ & & VAS (0 - 10 points) & & & & M5: -7.08 to -4.27 , \\
\hline & IG: 22.8 & pSWD: $3 x / w k$ for & & & & & $\mathrm{p}=0.0 \S$ \\
\hline & CG: 22.9 & $\begin{array}{l}15 \text { min on lumbar } \\
\text { spine, no heat }\end{array}$ & & & & & $\begin{array}{l}\text { M6: }-8.17 \text { to }-5.68 \\
p=0.0 \$\end{array}$ \\
\hline & $\begin{array}{l}\text { Symptomatic (mo): } \\
>3\end{array}$ & production & & & & & \\
\hline & & $\begin{array}{l}\text { EP: therapeutic } \\
\text { exercises, ADL } \\
\text { instructions not } \\
\text { further specified }\end{array}$ & & & & & \\
\hline & & $\begin{array}{l}\text { NSAID: } \\
\text { Meloxicam, } \\
15 \mathrm{mg} / \mathrm{d}\end{array}$ & & & & & \\
\hline \multicolumn{2}{|c|}{ Ansari et al.Iran } & IG: US & Extension/ & Baseline (M0) & Extension ROM & & \\
\hline (2006) & $\mathrm{N}=15$ & CG: pUS & flexion/right/left & After $5^{\text {th }}$ session (M1) & US (IG, N = 5) & pUS (CG, N = 5) & Sign. IG vs CG \\
\hline & DO: $N=5$ & & lateral flexion ROM & After $10^{\text {th }}$ session (M2) & $\mathrm{M} 0: 23.8 \pm 4.15$ & M0: $27.2 \pm 3.03$ & M1-M2: $p=0.0165$ \\
\hline \multirow{25}{*}{$\begin{array}{l}\text { RCT } \\
\text { PEDro- } \\
\text { score: } \\
5 / 10\end{array}$} & & Time: 10 sessions, & $\left({ }^{\circ}\right)$ & & $M 1: 26.6 \pm 4.7$ & M1: $30.2 \pm 3.2$ & \\
\hline & $\begin{array}{l}\text { IG: } N=5 \\
\text { Male: } N=5 \\
\text { Female: } N=0\end{array}$ & $\begin{array}{l}3 \mathrm{x} / \mathrm{wk} \\
\text { US mod.: } \\
\text { continuous }\end{array}$ & FRI & No FU & $\begin{array}{l}\text { M2: } 30 \pm 6.4 \\
\text { IG*time M0-M2: } \\
\mathrm{p}=0.04 \S \\
\text { Flexion ROM }\end{array}$ & $\begin{array}{l}\text { M2: } 29 \pm 4.2 \\
\text { IG*time M0-M2: } \\
p>0.05\end{array}$ & \\
\hline & CG: $N=5$ & intensity 1.5 & & & US (IG, N = 5) & pUS (CG, N = 5) & Sign. \\
\hline & Male: $\mathrm{N}=2$ & $\mathrm{~W} / \mathrm{cm}^{2}$, frequency 1 & & & M0: $117.4 \pm 2.5$ & M0: $103.4 \pm 13.39$ & $p>0.05$ \\
\hline & Female: $\mathrm{N}=3$ & $\begin{array}{l}\text { MHz, ERA } 5 \mathrm{~cm}^{2} \text {, } \\
\text { lumbar back }\end{array}$ & & & $\begin{array}{l}\text { M1: } 118.8 \pm 11.3 \\
\text { M2: } 128.6 \pm 14.3\end{array}$ & $\begin{array}{l}\text { M1: } 107 \pm 22.1 \\
\text { M2: } 109.2 \pm 10.6\end{array}$ & \\
\hline & Age (y): & region, duration & & & Right lateral flexion ROM & & \\
\hline & IG: $35.5 \pm 13.8$ & calculated by Gray's & & & US $(I G, N=5)$ & pUS (CG, N = 5) & Sign. IG vs CG \\
\hline & CG: $26.4 \pm 11.26$ & formula & & & $\begin{array}{l}\text { M0: } 20.2 \pm 7.3 \\
\text { M1: } 24.8 \pm 6.2\end{array}$ & $\begin{array}{l}\text { M0: } 22.6 \pm 5.2 \\
\text { M1: } 25.8 \pm 4.9\end{array}$ & M1-M2: $p=0.032 s$ \\
\hline & BMI $\left(\mathrm{kg} / \mathrm{m}^{2}\right):$ & & & & M2: $28 \pm 8.6$ & M2: $26 \pm 4.8$ & \\
\hline & IG: $28.33 \pm 2.9$ & & & & IG*time M0-M2: & CG*time M0-M2: & \\
\hline & CG: $23.8 \pm 5.2$ & & & & $\begin{array}{l}\mathrm{p}=0.04 \mathrm{~s} \\
\text { Left lateral flexion ROM }\end{array}$ & $p>0.05$ & \\
\hline & Symptomatic (mo): & & & & US $(I G, N=5)$ & pUS (CG, N = 5) & Sign. \\
\hline & IG: $13.8 \pm 9.8$ & & & & M0: $22 \pm 7.77$ & M0: $23.8 \pm 6.53$ & $\mathrm{n} / \mathrm{a}$ \\
\hline & CG: $14.8 \pm 10.7$ & & & & M1: $25.8 \pm 4.7$ & M1: $27.8 \pm 4.3$ & \\
\hline & & & & & M2: $28 \pm 7.1$ & $M 2: 28.4 \pm 4$ & \\
\hline & & & & & $\begin{array}{l}\mathrm{IG}^{*} \text { time } \mathrm{M} 0-\mathrm{M} 2: \\
\mathrm{p}=0.04 \S\end{array}$ & $\begin{array}{l}\text { CG*time M0-M2: } \\
p>0.05\end{array}$ & \\
\hline & & & & & FRI & & \\
\hline & & & & & US (IG, N = 5) & pUS (CG, N = 5) & Sign. \\
\hline & & & & & M0: $56.5 \pm 20.35$ & M0: $46.95 \pm 14.38$ & $\mathrm{n} / \mathrm{a}$ \\
\hline & & & & & M1: $45.15 \pm 14.5$ & M1: $41.6 \pm 15.9$ & \\
\hline & & & & & M2: $34.5 \pm 13.5$ & $M 2: 39.9 \pm 16.5$ & \\
\hline & & & & & IG*time M0-M2: & CG*time M0-M2: & \\
\hline & & & & & $\mathrm{p}=0.042^{\S}$ & $\mathrm{p}=0.043^{\S}$ & \\
\hline & & & & & $\begin{array}{l}\text { IG*time M0-M1: } \\
p>0.05\end{array}$ & $\begin{array}{l}\text { CG*time M0-M1: } \\
p>0.05\end{array}$ & \\
\hline & & & & & $\begin{array}{l}\mathrm{IG}^{*} \text { time M1-M2 } \\
\text { to } \mathrm{M} 2: \mathrm{p}=0.016^{\S}\end{array}$ & $\begin{array}{l}\text { CG*time M1-M2 to } \\
\text { M2: } p=0.032^{\S}\end{array}$ & \\
\hline \multirow{3}{*}{$\begin{array}{l}\text { Durmus et } \\
\text { al. (2010) }\end{array}$} & Turkey & IG: US + EP & Lumbar Schober & Baseline (M0) & Lumbar Schober & & \\
\hline & $N=68$ & CG: EP & $(\mathrm{cm})$ & $6^{\text {th }}$ wk (M1) & US + EP (IG, N = 19) & EP (CG, $N=20)$ & Sign. IGs vs CG \\
\hline & Male: $\mathrm{N}=\mathrm{n} / \mathrm{a}$ & & & & M0: $14.26 \pm 0.88$ & M0: $14.55 \pm 0.74$ & $p=0.614$ \\
\hline \multirow[t]{2}{*}{ RCT } & Female: $\mathrm{N}=\mathrm{n} / \mathrm{a}$ & Time: $6 \mathrm{wk}, 3 \mathrm{~d} / \mathrm{wk}$ & Modified lumbar & No FU & M1: $14.58 \pm 0.94$ & $M 1: 14.80 \pm 0.93$ & \\
\hline & DO: $N=9$ & & Schober $(\mathrm{cm})$ & & $\mathrm{IG}^{*}$ time: $\mathrm{p}=0.01 \S$ & $\mathrm{CG}^{*}$ time: $\mathrm{p}=0.029 \S$ & \\
\hline \multirow{11}{*}{$\begin{array}{l}\text { PEDro- } \\
\text { score: } \\
6 / 10\end{array}$} & & US mod.: & & & Modified lumbar Schober & & \\
\hline & IG: $N=19$ & continuous & Fingertip to floor & & US + EP (IG, N = 19) & EP $(C G, N=20)$ & Sign. IG vs CG \\
\hline & CG: $N=20$ & intensity $1 \mathrm{~W} / \mathrm{cm}^{2}$, & distance $(\mathrm{cm})$ & & M0: $21.57 \pm 1.42$ & M0: $21.55 \pm 1.31$ & $p=0.930$ \\
\hline & Age (y): & ERA $4 \mathrm{~cm}^{2}$, & BDI & & $\mathrm{IG}^{*}$ time: $\mathrm{p}=0.001^{\S}$ & CG*time: $p=0.002^{\S}$ & \\
\hline & IG: $48.31 \pm 8.95$ & paravertebral & & & Fingertip to floor distance & (median min-max) & \\
\hline & CG: $47.05 \pm 12.46$ & low back region, & ODQ (\%) & & US + EP (IG, N = 19) & EP $(C G, N=20)$ & Sign. IG vs CG \\
\hline & & $10 \mathrm{~min}$ & & & M0: $1(0-23)$ & M0: $0(0-24)$ & $p=0.850$ \\
\hline & BMI $\left(\mathrm{kg} / \mathrm{m}^{2}\right):$ & & PDI & & M1: $0(0-23)$ & M1: $0(0-14)$ & \\
\hline & IG: $28.89 \pm 3.98$ & EP: supervised, & & & IG*time: $p=0.009 \S$ & CG*time: $p=0.11$ & \\
\hline & CG: $28.50 \pm 1.84$ & group-exercise & & & BDI $\mathrm{r}-2-5$ & & \\
\hline & & program, $3 \mathrm{~d} / \mathrm{w}, 45$ & & & US + EP (IG, N = 19) & EP (CG, N = 20) & Sign. IG vs CG \\
\hline
\end{tabular}


S. Wittenwiler et al.

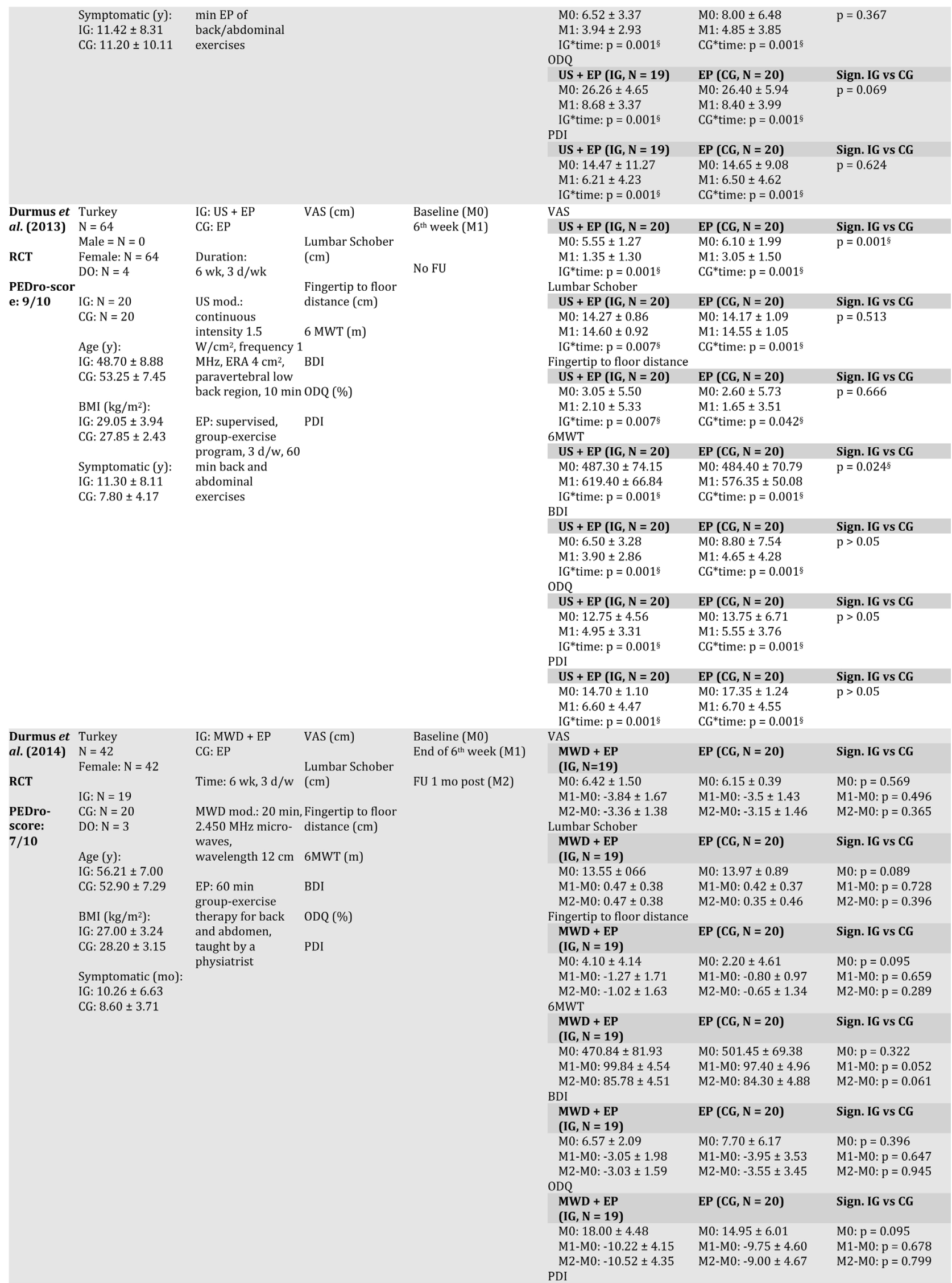




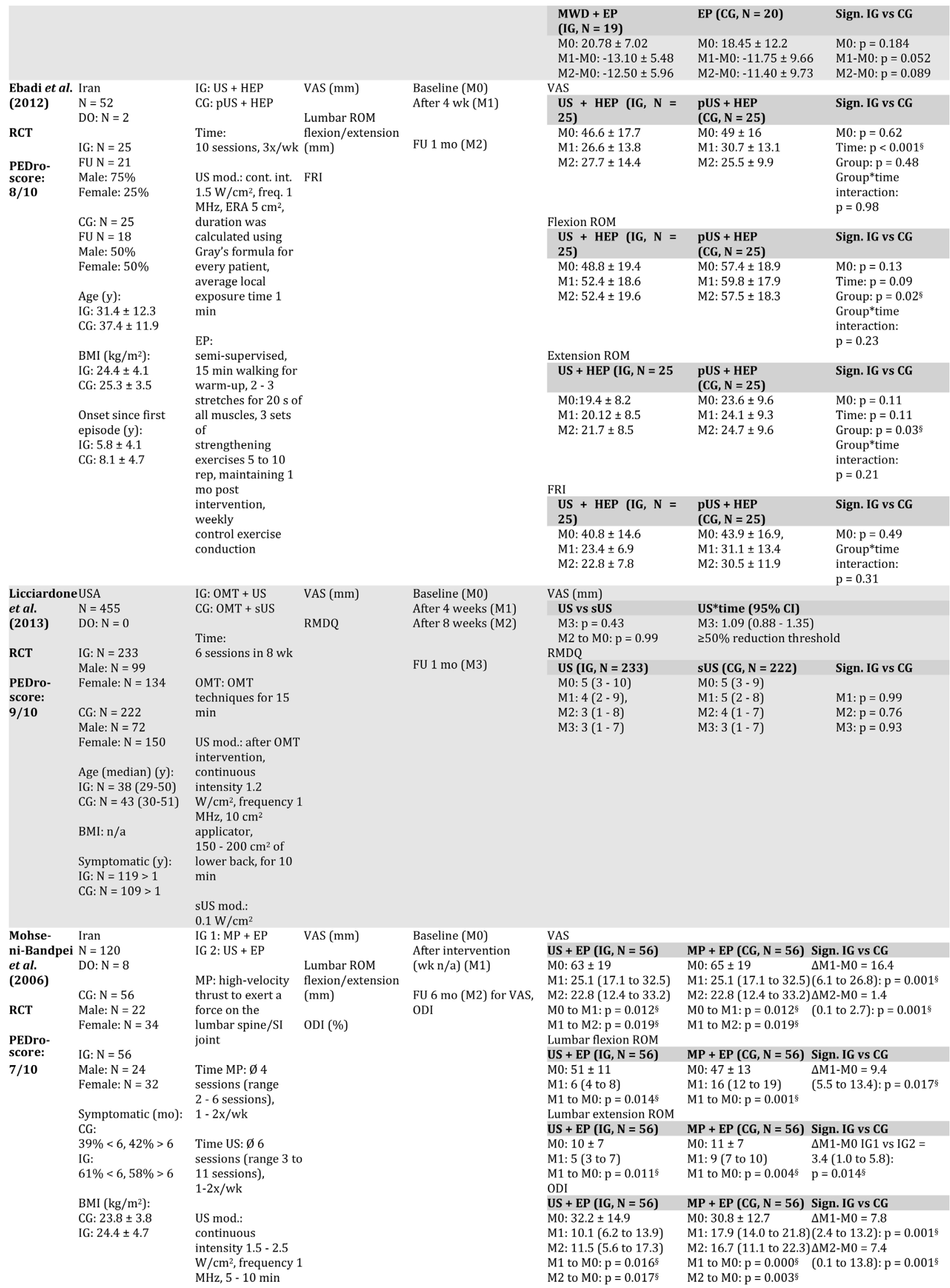




\begin{tabular}{|c|c|c|c|c|c|c|}
\hline \multirow{3}{*}{$\begin{array}{l}\text { Shakoor et } \\
\text { al. (2008) }\end{array}$} & \multirow{3}{*}{$\begin{array}{l}\text { Bangladesh } \\
\mathrm{N}=127\end{array}$} & \multicolumn{5}{|l|}{$\begin{array}{l}\text { EP: written, } \\
\text { individual per } \\
\text { patient, generated } \\
\text { by "PhysioTools" } \\
\text { computer package }\end{array}$} \\
\hline & & IG: $\quad$ VAS & Baseline (M0) & Sum of Lattinnen's $t$ & core and VAS $(0-30 \mathrm{pc}$ & ints) \\
\hline & & SWD + NSAID + EP Lattinnen's test & End of W1 (M1) & SWD (IG, N = n/a) & pSWD (CG, N = n/a) & Sign. IG vs CG \\
\hline & DO: $\mathrm{N}=25$ & CG: $\quad$ score & End of W2 (M2) & M0: $15.16 \pm 3.01$ & M0: $15.64 \pm 3.25$ & M0: -1.53 to -0.92 , \\
\hline \multirow[t]{2}{*}{ RCT } & & pSWD + NSAID + EP & End of W3 (M3) & M1: $13.94 \pm 2.65$ & M1: $14.52 \pm 3.16$ & $\mathrm{p}=0.62$ \\
\hline & $N=102$ & & End of W4 (M4) & M2: $11.86 \pm 2.40$ & $M 2: 12.42 \pm 3.41$ & M1: -1.72 to 0.56 , \\
\hline \multirow{9}{*}{$\begin{array}{l}\text { PEDro- } \\
\text { score: } \\
\mathbf{5 / 1 0}\end{array}$} & Male: $\mathrm{N}=42$ & SWD mod.: 27.33 & End of W5 (M5) & M3: $11.02 \pm 2.77$ & M3: $12.19 \pm 3.32$ & $\mathrm{p}=0.31$ \\
\hline & Female: $\mathrm{N}=60$ & $\mathrm{~Hz}, 11 \mathrm{~m}$ & End of W6 (M6) & M4: $10.30 \pm 2.56$ & M4: $11.77 \pm 3.61$ & M2: -1.72 to 0.59 , \\
\hline & Age (y): & $\begin{array}{l}\text { wavelength, } 15 \mathrm{~min} \\
3 \mathrm{x} / \mathrm{w}, 6 \mathrm{wk}\end{array}$ & & $\begin{array}{l}\text { M5: } 9.66 \text { V } 2.55 \\
\text { M6: } 9.04 \pm 2.49\end{array}$ & $\begin{array}{l}\text { M5: } 11.60 \pm 3.93 \\
\text { M6: } 11.48 \pm 4.02\end{array}$ & $\begin{array}{l}\mathrm{p}=0.33 \\
\text { M3: }-2.27 \text { to }-0.02 \text {, }\end{array}$ \\
\hline & IG: $44.50 \pm 8.94$ & & No FU & IG*time: $p=0.0 \$$ & $\mathrm{CG}^{*}$ time: $\mathrm{p}=0.0^{\S}$ & $\mathrm{p}=0.05 \$$ \\
\hline & CG: $40.02 \pm 6.49$ & $\begin{array}{l}\text { NSAID: Naproxen } \\
250 \mathrm{mg} 2 \mathrm{x} / \mathrm{d}\end{array}$ & & & & $\begin{array}{l}\text { M4: }-2.60 \text { to } 0.24 \\
p=0.02^{\S}\end{array}$ \\
\hline & $\operatorname{BMI}^{\#}\left(\mathrm{~kg} / \mathrm{m}^{2}\right):$ & & & & & M5: -3.23 to -0.63 , \\
\hline & IG: 25.02 & & & & & $\mathrm{p}=0.00 \S$ \\
\hline & CG: 24.82 & 10 reps, $2 x / d, 6$ wk & & & & $\begin{array}{l}\text { M6: }-3.75 \text { to }-1.12, \\
p=0.00 \text { s }\end{array}$ \\
\hline & $\begin{array}{l}\text { Symptomatic (mo): } \\
\text { IG: } 40.20 \pm 35.47 \\
\text { CG: } 35.24 \pm 40.12\end{array}$ & & & & & \\
\hline
\end{tabular}

Figure 4. Detailed overview on the results of the included studies. Legend. BDI = back depression index, $\mathrm{BMI}^{(\#)}=\mathrm{body}^{\mathrm{mass}}$ in$\mathrm{dex}^{\text {(self-calculated weighted means) }}, \mathrm{CG}=$ control group, $\mathrm{DO}=$ drop outs, $(\mathrm{H}) \mathrm{EP}=($ home$)$ exercise program, ERA = effective radiating area, $\mathrm{FRI}=$ functional rating index, $\mathrm{FU}=$ follow-up, $\mathrm{IG}=$ intervention group, Meth. = methodological, $\mathrm{MP}=$ manipulation, mod. $=$ modality, MWD = microwave diathermy, $6 \mathrm{MWT}=6$-min walk test, $\mathrm{N}=$ number of participants, $\mathrm{n} / \mathrm{a}=$ not applicable, $\mathrm{ODQ}=$ Oswestry Disability questionnaire, $\mathrm{p}=$ placebo, $\mathrm{PDI}=$ pain disability index, $\mathrm{RMDQ}=$ Roland-Morris disability questionnaire, $\mathrm{ROM}=$ range of motion, $\mathrm{s}=$ sham, SF-36 = medical outcomes study short form-36 health survey, $\mathrm{SI}=$ sacro-iliac, $\mathrm{SWD}=$ short-wave diathermy, US $=$ ultrasound, VAS $=$ visual analog scale, ${ }^{\varsigma}=$ significant at alpha $<0.05,{ }^{*}=$ within-group results over time.

distribution was not mentioned by $\mathrm{n}=3$ studies [29] [30] [31], $\mathrm{n}=2$ studies were conducted with female participants only [30] [36], and $n=4$ studies included male and female participants [28] [32] [34] [35]. Age, BMI (except one) [32] and symptomatic back pain time before enrolment could be extracted from all studies. Treatment sessions ranged from 2 to 18 sessions over 4 to 8 weeks. Four of the included studies did a follow-up measurement, out of them $n=3$ one month after the end of treatment [31] [32] [36] and $n=1$ after six months post treatment [33].

The studies comprised a wide range of assessment tools to rate the outcome parameters: pain was assessed by the visual analog scale (VAS) [30] [31] [32] [33] [34] [36], Lattinnen's test score [34] [35] and tenderness score [34]. Functional parameters were assessed by trunk mobility (Range of Motion (ROM) [28] [30] [36], (modified) lumbar Schober test [29] [30] [36], fingertip to floor distance [29] [30] [36], trunk muscle strength [30], endurance [30] [31] [36], activation [28] [33], 6-min Walk Test (6MWT)) [30] [36], and questionnaires (Functional Rating Index (FRI) [28] [31], Pain Disability Index (PDI) [29] [30] [36], Oswestry Disability Questionnaire (ODQ) [29] [30] [33] [36], Back Depression Index (BDI) [36], Medical Outcomes Study Short Form-36 Health survey general health scale (SF-36 GH) [32] [36], Roland-Morris Disability Questionnaire (RMDQ)) [32].

\subsection{Treatment Effects on Outcome Parameters}

Out of $n=6$ studies on ultrasound treatment [28]-[33] a significant within and 
between-group pain reduction was reported by $\mathrm{n}=2$ studies, compared to exercise training alone $(p=0.012)[30]$ or to manipulation treatment $(p=0.001)$ [33]. Trunk mobility was mentioned in $\mathrm{n}=6$ studies [28] [29] [30] [31] [33] [36] of which all reported flexion mobility with $\mathrm{n}=1$ study including lateral flexion [28] and $n=3$ studies evaluating extension mobility [28] [31] [33]. Three studies reported significant within-group ROM improvements in flexion [29] [30] [33] for both the intervention and the control group. None of the $n=6$ studies showed significant between-group results. Out of the $\mathrm{n}=3$ studies on extension ROM, $n=2$ showed significant within-group results [28] [33], none of them found significant between-group results in favour of ultrasound treatment compared to placebo ultrasound treatment [28] or manipulation treatment [33]. Right lateral flexion ROM increased significantly within and between the placebo and ultrasound group $(\mathrm{p}=0.04, \mathrm{p}=0.032$, respectively), whereas left lateral flexion ROM significantly improved within-group $(\mathrm{p}=0.04)$ [28]. 6MWT results, evaluated by $\mathrm{n}=1$ study, were significant for within and between-groups ( $\mathrm{p}=0.001, \mathrm{p}=0.024$, respectively) [30]. Questionnaire outcomes were used in $\mathrm{n}=$ 6 studies [28]-[33]. The FRI score was significant within the placebo and treatment groups, no data on between-group results were mentioned [28]. The time $(\mathrm{p}<0.001)$ and group $(\mathrm{p}=0.004)$ main effects for the ultrasound treatment compared to the placebo intervention were significant in Ebadi et al. (2012), with a non-significant group ${ }^{*}$ time interaction $(\mathrm{p}=0.31)$ [31]. The PDI, ODQ and BDI showed significant within-group results $(p=0.001)$ for the combined ultrasound and exercise treatment group and exercise group alone and non-significant between group results [29] [30]. Mohseni-Bandpei et al. (2006) reported significant within-group results for the ultrasound group and manipulative control group and significant between-group results $(p=0.001)$ in favour of manipulation treatment [33]. The SF-36 subscale items showed significant within-group differences for all items $(p=0.001)$ [29] [30] with significant between-group results for "physical function" ( $p=0.009)$ [29], "social function" ( $p=$ 0.016 ) [29] and "physical function" ( $p=0.021)$ [30], "pain" ( $p=0.046)$ [30] and "energy" ( $\mathrm{p}=0.009)$ [30] compared to exercise treatment alone. Licciardone et al. (2013) mentioned non-significantly different total SF-36 scores $(\mathrm{p}=0.53)$ and RMDQ outcomes $(\mathrm{p}=0.76)$ between ultrasound treatment and placebo intervention (eAddenda Appendix III) [29] [32].

Three studies focused on diathermy treatment, $\mathrm{n}=2$ studies on short-wave diathermy [34] [35] and $n=1$ on microwave diathermy [36]. For short-wave diathermy treatment, significant results in pain reduction were mentioned compared to the placebo group $(\mathrm{p}=0.0)$ [34] [35]. Shakoor et al. (2008) reported significant within-group results at all time intervals for both the intervention and the placebo group [35]. Non-significant between-group results for microwave diathermy combined with exercise versus exercise alone $(p=0.496)$ were mentioned [36]. Trunk mobility in flexion showed no significant difference between the microwave diathermy and exercise group compared to the exercise 
group alone $(\mathrm{p}>0.05)$ [36], as well as for 6MWT performance and all reported questionnaire outcomes $(\mathrm{p}>0.05)$ [36].

\section{Discussion}

The aim of this systematic review was to assess the evidence for the use of thermal therapy in NSCLBP patients on pain and physical outcome parameters and to evaluate the methodological quality of the corresponding studies.

\subsection{Evidence for Thermal Therapy}

Despite the search strategy no studies on heat wraps, hot or mud packs fulfilled the inclusion criteria. To evaluate solely the thermal effect of local interventions, unbiased by water pressure or whole-body thermal applications and its physiological reactions, balneo/spa therapy was deliberately omitted as a keyword. However, several clinical trials showed positive short and long-term effects for balneo therapy in CLBP patients on pain, quality of life and physical function [37] [38].

The findings of this review, for a fraction of electrophysical agents only (continuous ultrasound, short-wave diathermy and microwave diathermy), was that all observed treatment interventions except microwave diathermy [36] significantly improved pain symptoms, with only two studies documenting the intake of oral pain killers [34] [35]. The ineffectiveness of microwave diathermy on pain relief is supported by a study on non-specific chronic neck pain patients [39]. The significant improvement in pain between the groups was underpinned by the SF-36 subscale items physical function, pain and energy [30]. The SF-36 subscale items "physical function, social function, energy" may be positively affected by thermal therapy applied by means of electrophysical agents, of which ultrasound treatment may be superior to diathermy application. Surprisingly, mental health and general health were unaffected by pain reduction [30]. However, control group results showed significant pain reduction over time, limiting the positive effect of any electrophysical treatment for NSCLBP on pain. Supporting this finding, Koldas et al. (2008) reported significant improvements in global health and disability questionnaire results rather than pain reduction for physical therapy intervention group (hot pack, ultrasound and TENS) compared to home exercise alone [40].

The included studies demonstrated that trunk mobility in the frontal and sagittal planes was indeed reduced in NSCBLP patients but improved independently of treatment, supporting the guidelines' recommendations to remain physically active, rather than applying thermal therapy [12] [41]. Additionally, NSCLBP patients attained walking distances comparable to healthy subjects [42], assuming only a marginal negative effect of NSCLBP on walking distance performance and questioning the efficacy of the 6MWT distance as sensitive outcome parameter for NSCLBP. Earlier literature has reported that all forms of therapy have shown a positive effect on depression and global health [43]. This was sup- 
ported by the questionnaire score results of this review. Moreover, Kurklinsky et al. (2016) challenged pain reduction in chronic pain patients proposing aiming at functional and quality of life improvements instead [44].

All ultrasound studies applied continuous mode to ensure a thermal effect. Unfortunately, there was a wide heterogeneity in the reported ultrasound settings, although existing literature on ultrasound modalities and corresponding heat rates in specific depths for muscle and connective tissue exist [45] [46] [47] [48]. The participants' characteristic between the studies was heterogenic in terms of being symptomatic with NSCLBP for a period ranging from 3 months to 12 years. To presume that the duration of symptoms may negatively affect treatment outcomes cannot be supported since even the control groups showed improvement in the assessed outcome parameters.

\subsection{Methodological Quality}

The mean methodological quality of the included studies did not attain the total PEDro scores proposed by Moseley et al. (2011) [49]. Higher total PEDro scores would have been achievable through thorough reporting of statistical procedures and resolute blinding of personnel and/or outcome assessors [49] [50]. However, the methodological quality did not affect the studies' outcomes.

\subsection{Limitations and Future Research}

Figure 2 and Figure 3 depict that the included studies had a high risk of bias for blinding personnel and outcome assessors and an unclear risk of bias for random sequence generation, allocation concealment and other bias. The risk of a selection bias could be reduced by consistent reporting of random sequence generation and allocation concealment. Depending on the chosen thermal therapy treatment blinding of participants may be impossible due to the warming effect, nevertheless, $>50 \%$ low risk of bias was achieved. Surprisingly, as accessible, is the high risk of performance, detection bias and attrition bias, since hardly any follow-up measurements were conducted. Future studies are recommended to specify their study protocols and procedures to further reduce the risk of selection, reporting and other bias. Blinding of outcome assessors should become a matter of course, blinding of participants and personnel highly advised wherever possible to ensure a low risk of performance and detection bias. Further, there is a lack of studies using hot packs or heat wraps as applications method, although commonly utilized by patients at home.

\section{Conclusion}

In line with previous findings [16] [17], this review included recently published studies reaffirmed the contradictory evidence for thermal therapy on NSCLBP. Only electrophysical treatment methods fulfilled the inclusion criteria. Little evidence for direct post treatment pain reduction exists for continuous ultrasound treatment of 10 minutes' duration, three days per week for 6 weeks, and 
short-wave diathermy treatment of 15 minutes' duration, three days per week for 2 to 3 weeks. This short-term pain reduction achieved may be utilized as preparation for physical activity and exercising [15] [51]. Remaining physically active and attending exercising are the main statements of international guidelines for chronic low back pain. Nevertheless, with respect to the complexity of neurophysiological mechanisms behind chronic pain syndromes, individual therapy and advice should be sought [52]. Although the effect of thermal therapy has been described in literature [19], as applied by means of electrophysical agents (continuous ultrasound, short-wave and microwave diathermy), the authors conclude that there is only marginal evidence to support its application in patients with NSCLBP.

\section{Acknowledgements}

The authors thank Mrs. Lindsay Melcher-Kunz for providing language help and proof reading of the manuscript, and the "Thim van der Laan foundation" for the financial support.

\section{Conflict of Interest}

The authors declare no conflict of interest.

\section{Author Contributions}

Conceived and designed the experiments: SW, RS, RC. Data extraction and quality assessment: SW, RS, EH. Risk of bias assessment: SW, RS, EH. Analysis of the data: SW, RS. Wrote the paper: SW, RS. Read and approved final version of manuscript: SW, RS, EH, RC.

\section{References}

[1] Gureje, O., Von Korff, M., Simon, G. and Gater, R. (1998) Persistent Pain and Well-Being: A World Health Organization Study in Primary Care. JAMA, 280, 147-151.

[2] Merskey, H. and Bogduk, N. (1986) Classification of Chronic Pain. Descriptions of Chronic Pain Syndromes and Definitions of Pain Terms. International Association for the Study of Pain, Subcommittee on Taxonomy, 3, S1-226.

[3] Freburger, J.K., Holmes, G.M., Agans, R.P., Jackman, A.M., Darter, J.D., Wallace, A.S., Castel, L.D., Kalsbeek, W.D. and Carey, T.S. (2009) The Rising Prevalence of Chronic Low Back Pain. Archives of Internal Medicine, 169, 251-258.

https://doi.org/10.1001/archinternmed.2008.543

[4] Andrew, R., Derry, S., Taylor, R.S., Straube, S. and Phillips, C.J. (2014) The Costs and Consequences of Adequately Managed Chronic Non-Cancer Pain and Chronic Neuropathic Pain. Pain Practice, 14, 79-94. https://doi.org/10.1111/papr.12050

[5] Asche, C.V., Kirkness, C.S., McAdam-Marx, C. and Fritz, J.M. (2007) The Societal Costs of Low Back Pain: Data Published between 2001 and 2007. Journal of Pain \& Palliative Care Pharmacotherapy, 21, 25-33. https://doi.org/10.1080/J354v21n04_06

[6] Dagenais, S., Caro, J. and Haldeman, S. (2008) A Systematic Review of Low Back Pain Cost of Illness Studies in the United States and Internationally. The Spine 
Journal, 8, 8-20. https://doi.org/10.1016/j.spinee.2007.10.005

[7] Wenig, C.M., Schmidt, C.O., Kohlmann, T. and Schweikert, B. (2009) Costs of Back Pain in Germany. European Journal of Pain, 13, 280-286. https://doi.org/10.1016/j.ejpain.2008.04.005

[8] Hildebrandt, J. (2004) Gibt es einen unspezifischen Rückenschmerz? Zeitschrift Für Orthopädie Und Ihre Grenzgebiete, 142, 139-145. https://doi.org/10.1055/s-2004-822623

[9] Krismer, M. and van Tulder, M. (2007) Strategies for Prevention and Management of Musculoskeletal Conditions. Low Back Pain (Non-Specific). Best Practice \& Research: Clinical Rheumatology, 21, 77-91. https://doi.org/10.1016/j.berh.2006.08.004

[10] Elzahaf, R.A., Tashani, O.A., Unsworth, B.A. and Johnson, M.I. (2012) The Prevalence of Chronic Pain with an Analysis of Countries with a Human Development Index Less than 0.9: A Systematic Review without Meta-Analysis. Current Medical Research and Opinion, 28, 1221-1229. https://doi.org/10.1185/03007995.2012.703132

[11] Koes, B.W., van Tulder, M., Lin, C.W., Macedo, L.G., McAuley, J. and Maher, C. (2010) An Updated Overview of Clinical Guidelines for the Management of Non-Specific Low Back Pain in Primary Care. European Spine Journal, 19, 2075-2094. https://doi.org/10.1007/s00586-010-1502-y

[12] Bekkering, G.E., van Tulder, M.W., Hendriks, E.J., Koopmanschap, M.A., Knol, D.L., Bouter, L.M. and Oostendorp, R.A. (2005) Implementation of Clinical Guidelines on Physical Therapy for Patients with Low Back Pain: A Randomized Trial Comparing Patient Outcomes after a Standard and Active Implementation Strategy. Physical Therapy, 85, 544-555.

[13] Liddle, S.D., Gracey, J.H. and Baxter, G.D. (2007) Advice for the Management of Low Back Pain: A Systematic Review of Randomised Controlled Trials. Manual Therapy, 12, 310-327. https://doi.org/10.1016/j.math.2006.12.009

[14] Poitras, S. and Brosseau, L. (2008) Evidence-Informed Management of Chronic Low Back Pain with Transcutaneous Electrical Nerve Stimulation, Interferential Current, Electrical Muscle Stimulation, Ultrasound, and Thermotherapy. The Spine Journal, 8, 226-233.

[15] Allen, R.J. (2006) Physical Agents Used in the Management of Chronic Pain by Physical Therapists. Physical Medicine \& Rehabilitation Clinics of North America, 17, 315-345. https://doi.org/10.1016/j.pmr.2005.12.007

[16] French, S.D., Cameron, M., Walker, B.F., Reggars, J.W. and Esterman, A.J. (2006) Superficial Heat or Cold for Low Back Pain. The Cochrane Database of Systematic Reviews, No. 1, CD004750. https://doi.org/10.1002/14651858.CD004750

[17] Airaksinen, O., Brox, J.I., Cedraschi, C., Hildebrandt, J., Klaber-Moffett, J., Kovacs, F., Mannion, A.F., Reis, S., Staal, J.B., Ursin, H., Zanolin, G. and COST B13 Working Group on Guidelines for Chronic Low Back Pain (2006) Chapter 4. European Guidelines for the Management of Chronic Nonspecific Low Back Pain. European Spine Journal, 15, S192-S300. https://doi.org/10.1007/s00586-006-1072-1

[18] Crowe, M., Whitehead, L., Jo Gagan, M., Baxter, D. and Panckhurst, A. (2010) Self-Management and Chronic Low Back Pain: A Qualitative Study. Journal of Advanced Nursing, 66, 1478-1486. https://doi.org/10.1111/j.1365-2648.2010.05316.x

[19] Nadler, S.F., Weingand, K. and Kruse, R.J. (2004) The Physiological Basis and Clinical Applications of Cryotherapy and Thermotherapy for the Pain Practitioner. Pain Physician, 7, 395-399. 
[20] Davis, K.D., Kwan, C.L., Crawley, A.P. and Mikulis, D.J. (1998) Functional MRI Study of Thalamic and Cortical Activations Evoked by Cutaneous Heat, Cold, and Tactile Stimuli. Journal of Neurophysiology, 80, 1533-1546. https://doi.org/10.1152/jn.1998.80.3.1533

[21] Mulkern, R., McDannold, N., Hynynen, K., Fielding, J., Panych, L., Jolesz, F.A. and Weingand, K. (1999) Temperature Distribution Change in Low Back Muscles during Applied Topical Heat: A Magnetic Resonance Thermometry Study. Proceedings of the International Society for Magnetic Resonance in Medicine, Philadelphia, 22-28 May 1999, 1054.

[22] Nadler, S.F., DePrince, M.L. and Stitik, T.P. (1999) Experimentally Induced Trapezius Fatigue and the Effects of Topical Heat on the EMG Power Density Spectrum. American Journal of Physical Medicine \& Rehabilitation, 80, 1123.

[23] Richardson, W.S., Wilson, M.C., Nishikawa, J. and Hayward, R.S. (1995) The Well-Built Clinical Question: A Key to Evidence-Based Decisions. ACP Journal Club, 123, A12-A13.

[24] Knobloch, K., Yoon, U. and Vogt, P.M. (2011) Preferred Reporting Items for Systematic Reviews and Meta-Analyses (PRISMA) Statement and Publication Bias. Journal of Cranio-Maxillo-Facial Surgery, 39, 91-92. https://doi.org/10.1016/j.jcms.2010.11.001

[25] de Morton, N.A. (2009) The PEDro Scale Is a Valid Measure of the Methodological Quality of Clinical Trials: A Demographic Study. The Australian Journal of Physiotherapy, 55, 129-133. https://doi.org/10.1016/S0004-9514(09)70043-1

[26] PEDro. Physiotherapy Evidence Database. Downloads, Statistics. http://www.pedro.org.au

[27] Higgins, J.P.T. and Green, S. (2011) Cochrane Handbook for Systematic Reviews of Interventions Version 5.1.0. The Cochrane Collaboration. https://handbook-5-1.cochrane.org

[28] Ansari, N.N., Ebadi, S., Talebian, S., Naghdi, S., Mazaheri, H., Olyaei, G. and Jalaie, S. (2006) A Randomized, Single Blind Placebo Controlled Clinical Trial on the Effect of Continuous Ultrasound on Low Back Pain. Electromyography and Clinical Neurophysiology, 46, 329-336.

[29] Durmus, D., Durmaz, Y. and Canturk, F. (2010) Effects of Therapeutic Ultrasound and Electrical Stimulation Program on Pain, Trunk Muscle Strength, Disability, Walking Performance, Quality of Life, and Depression in Patients with Low Back Pain: A Randomized-Controlled Trial. Rheumatology International, 30, 901-910. https://doi.org/10.1007/s00296-009-1072-7

[30] Durmus, D., Alayli, G., Goktepe, A.S., Taskaynatan, M.A., Bilgici, A. and Kuru, O. (2013) Is Phonophoresis Effective in the Treatment of Chronic Low Back Pain? A Single-Blind Randomized Controlled Trial. Rheumatology International, 33, 1737-1744. https://doi.org/10.1007/s00296-012-2634-7

[31] Ebadi, S., Ansari, N.N., Naghdi, S., Jalaei, S., Sadat, M., Bagheri, H., Vantulder, M.W., Henschke, N. and Fallah, E. (2012) The Effect of Continuous Ultrasound on Chronic Non-Specific Low Back Pain: A Single Blind Placebo-Controlled Randomized Trial. BMC Musculoskeletal Disorders, 13, 192.

https://doi.org/10.1186/1471-2474-13-192

[32] Licciardone, J.C., Minotti, D.E., Gatchel, R.J., Kearns, C.M. and Singh, K.P. (2013) Osteopathic Manual Treatment and Ultrasound Therapy for Chronic Low Back Pain: A Randomized Controlled Trial. The Annals of Family Medicine, 11, 122-129. https://doi.org/10.1370/afm.1468 
[33] Mohseni-Bandbei, M.A., Critchley, J., Staunton, T. and Richardson, B. (2006) A Prospective Randomised Controlled Trial of Spinal Manipulation and Ultrasound in the Treatment of Chronic Low Back Pain. Physiotherapy, 92, 34-42. https://doi.org/10.1016/j.physio.2005.05.005

[34] Ahmed, M.S., Shakoor, M.A. and Khan, A.A. (2009) Evaluation of the Effects of Shortwave Diathermy in Patients with Chronic Low Back Pain. Bangladesh Medical Research Council Bulletin, 35, 18-20. https://doi.org/10.3329/bmrcb.v35i1.2320

[35] Shakoor, M.A., Rahman, M.S. and Moyeenuzzaman, M. (2008) Effects of Deep Heat Therapy on the Patients with Chronic Low Back Pain. Mymensingh Medical Journal, 17, S32-S38.

[36] Durmus, D., Ulus, Y., Alayli, G., Akyol, Y., Bilgici, A., Yazicioglu, K. and Kuru, O. (2014) Does Microwave Diathermy Have an Effect on Clinical Parameters in Chronic Low Back Pain? A Randomized-Controlled Trial. Journal of Back and Musculoskeletal Rehabilitation, 27, 435-443. https://doi.org/10.3233/BMR-140464

[37] Constant, F., Guillemin, F., Collin, J.F. and Boulangé, M. (1998) Use of Spa Therapy to Improve the Quality of Life of Chronic Low Back Patients. Medical Care, 36, 1309-1314. https://doi.org/10.1097/00005650-199809000-00003

[38] Kesiktas, N., Karakas, S., Gun, K., Murat, S. and Uludag, M. (2012) Balneotherapy for Chronic Low Back Pain: A Randomized, Controlled Study. Rheumatology International, 32, 3193-3199. https://doi.org/10.1007/s00296-011-2163-9

[39] Andrade Ortega, J.A., Cerón Fernández, E., García Llorent, R., Ribeiro González, M. and Delgado Martínez, A.D. (2014) Microwave Diathermy for Treating Nonspecific Chronic Neck Pain: A Randomized Controlled Trial. The Spine Journal, 14, 1712-1721. https://doi.org/10.1016/j.spinee.2013.10.025

[40] Koldas Dogan, S., Sonel Tur, B., Kurtais, Y. and Atay, M.B. (2008) Comparison of Three Different Approaches in the Treatment of Chronic Low Back Pain. Clinical Rheumatology, 27, 873-881. https://doi.org/10.1007/s10067-007-0815-7

[41] Qaseem, A., Wilt, T.J., McLean, R.M. and Forciea, M.A. (2017) Noninvasive Treatments for Acute, Subacute, and Chronic Low Back Pain: A Clinical Practice Guideline from the American College of Physicians. Annals of Internal Medicine, 166, 514-530. https://doi.org/10.7326/M16-2367

[42] Breda, C.A., Rodacki, A.L., Leite, N., Homann, D., Goes, S.M. and Stefanello, J.M. (2013) Physical Activity Level and Physical Performance in the 6-Minute Walk Test in Women with Fibromialgia. Revista Brasileira de Reumatologia, 53, 276-281. https://doi.org/10.1590/S0482-50042013000300005

[43] Garbi Mde, O., Hortense, P., Gomez, R.R., da Silva Tde, C., Castanho, A.C. and Sousa, F.A. (2014) Pain Intensity, Disability and Depression in Individuals with Chronic Back Pain. Revista Latino-Americana De Enfermagem, 22, 569-575. https://doi.org/10.1590/0104-1169.3492.2453

[44] Kurklinsky, S., Perez, R.B., Lacayo, E.R. and Sletten, C.D. (2016) The Efficacy of Interdisciplinary Rehabilitation for Improving Function in People with Chronic Pain. Pain Research and Treatment, 2016, Article ID: 7217684. https://doi.org/10.1155/2016/7217684

[45] Draper, D.O. and Ricard, M.D. (1995) Rate of Temperature Decay in Human Muscle Following $3 \mathrm{MHz}$ Ultrasound: The Stretching Window Revealed. Journal of Athletic Training, 30, 304-307.

[46] Gallo, J.A., Draper, D.O., Brody, L.T. and Fellingham, G.W. (2004) A Comparison of Human Muscle Temperature Increases during 3-MHz Continuous and Pulsed Ultrasound with Equivalent Temporal Average Intensities. Journal of Orthopaedic 
\& Sports Physical Therapy, 34, 395-401. https://doi.org/10.2519/jospt.2004.34.7.395

[47] Draper, D.O., Castel, J.C. and Castel, D. (1995) Rate of Temperature Increase in Human Muscle during $1 \mathrm{MHz}$ and $3 \mathrm{MHz}$ Continuous Ultrasound. Journal of Orthopaedic \& Sports Physical Therapy, 22, 142-150.

https://doi.org/10.2519/jospt.1995.22.4.142

[48] Watson, T. (2017) Electrotherapy on the Web-Educational Resources for Practitioners, Students and Educators. http://www.electrotherapy.org

[49] Moseley, A.M., Herbert, R.D., Maher, C.G., Sherrington, C. and Elkins, M.R. (2011) Reported Quality of Randomized Controlled Trials of Physiotherapy Interventions Has Improved over Time. Journal of Clinical Epidemiology, 64, 594-601. https://doi.org/10.1016/j.jclinepi.2010.08.009

[50] Stoop, R., Clijsen, R., Leoni, D., Soldini, E., Castellini, G., Redaelli, V. and Barbero, M. (2017) Evolution of the Methodological Quality of Controlled Clinical Trials for Myofascial Trigger Point Treatments for the Period 1978-2015: A Systematic Review. Musculoskeletal Science and Practice, 30, 1-9. https://doi.org/10.1016/j.msksp.2017.04.009

[51] Gordon, R. and Bloxham, S. (2016) A Systematic Review of the Effects of Exercise and Physical Activity on Non-Specific Chronic Low Back Pain. Healthcare (Basel), 4, E22.

[52] Massé-Alarie, H. and Schneider, C. (2016) Revisiting the Corticomotor Plasticity in Low Back Pain: Challenges and Perspectives. Healthcare (BaseI), 4, E67. 


\section{Appendix I}

Table A1. Overview on the MeSH-Term proven keywords.

\begin{tabular}{cl}
\hline Keywords & MeSH-Term \\
\hline diathermy & yes \\
fango packs & no \\
heat application/wrap & no \\
hot packs & no \\
hyperemic cream/gel/ointment/products & no \\
hyperthermia induced & yes \\
low back pain & yes \\
mud packs & yes \\
thermal therapy & no \\
thermotherapy & no \\
topical hyperemic products & no \\
topical warming products & no \\
ultrasonic therapy & yes \\
warming products & no \\
warmth application & no \\
\hline
\end{tabular}

\section{Appendix II}

Table A2. PEDro-score of the included studies.

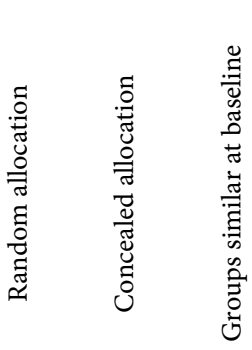

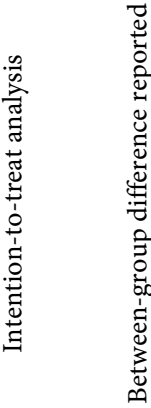

\begin{tabular}{|c|}
\hline 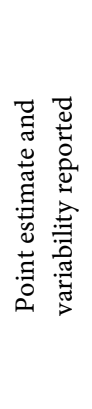 \\
\hline
\end{tabular}

\begin{tabular}{|c|c|c|c|c|c|c|c|c|c|c|c|}
\hline Ahmed et al. (2009) & - & - & + & - & - & - & + & - & + & + & 4 \\
\hline Ansari et al. (2006) & + & - & - & + & - & + & - & - & + & + & 5 \\
\hline Durmus et al. (2010) & + & - & + & + & - & + & - & - & + & + & 6 \\
\hline Durmus et al. (2013) & + & + & + & + & + & - & + & + & + & + & 9 \\
\hline Durmus et al. (2014) & + & + & + & - & + & - & + & - & + & + & 7 \\
\hline Ebadi et al. (2012) & + & + & + & + & - & - & + & + & + & + & 8 \\
\hline Licciardone et al. (2013) & + & + & + & + & - & + & + & + & + & + & 9 \\
\hline $\begin{array}{l}\text { Mohseni-Bandpei } \\
\text { et al. (2006) }\end{array}$ & + & + & + & - & - & + & + & + & + & + & 8 \\
\hline Shakoor et al. (2008) & + & + & + & - & - & - & - & - & + & + & 5 \\
\hline
\end{tabular}

Legend. $+=$ criteria fulfilled, $-=$ criteria not fulfilled. 


\section{Appendix III}

Table A3. Detailed overview on the SF-36 questionnaire results.

\begin{tabular}{|c|c|c|c|c|}
\hline Authors & SF-36 results & & & \\
\hline \multirow[t]{25}{*}{ Durmus et al. (2010) } & Subscale & US + EP (IG, N = 19) & $\mathrm{EP}(\mathrm{CG}, \mathrm{N}=20)$ & Significance IG vs CG \\
\hline & & M0: $75.0(35-85)$ & M0: $77.5(60-100)$ & \\
\hline & Physical function & M1: $90.0(65-100)$ & M1: $90.0(70-100)$ & $\mathrm{p}=0.009^{\S}$ \\
\hline & & $\mathrm{IG}^{\star}$ time: $\mathrm{p}=0.001^{\S}$ & $\mathrm{CG}^{*}$ time: $\mathrm{p}=0.001^{\S}$ & \\
\hline & & M0: $58.31 \pm 11.10$ & M0: $59.20 \pm 11.05$ & \\
\hline & Mental health & M1: $73.05 \pm 12.49$ & M1: $71.75 \pm 10.96$ & $\mathrm{p}=0.166$ \\
\hline & & $\mathrm{IG}^{\star}$ time: $\mathrm{p}=0.001^{\S}$ & $\mathrm{CG}^{\star}$ time: $\mathrm{p}=0.001^{\S}$ & \\
\hline & & M0: $44.0(44-88)$ & M0: $52.0(44-88)$ & \\
\hline & Pain & M1: 88.0 (66 - 99 & M1: $77.0(65-100)$ & $\mathrm{p}=0.285$ \\
\hline & & IG ${ }^{\star}$ time: $p=0.001^{\S}$ & $\mathrm{CG}^{\star}$ time: $\mathrm{p}=0.001^{\S}$ & \\
\hline & & M0: $51.05 \pm 15.32$ & M0: $52.75 \pm 17.53$ & \\
\hline & General health & M1: $65.52 \pm 16.90$ & M1: $64.25 \pm 15.99$ & $\mathrm{p}=0.231$ \\
\hline & & IG time: $p=0.001^{\S}$ & $C G^{\star}$ time: $\mathrm{p}=0.001^{\S}$ & \\
\hline & & M0: $55.0(44-88)$ & M0: $56.0(33-88)$ & \\
\hline & Social function & M1: $77.0(55-88)$ & M1: $77.0(44-88)$ & $\mathrm{p}=0.016^{\S}$ \\
\hline & & $\mathrm{IG}^{\star}$ time: $\mathrm{p}=0.001^{\S}$ & $\mathrm{CG}^{\star}$ time: $\mathrm{p}=0.001^{\S}$ & \\
\hline & & M0: $50.0(0-100)$ & M0: $62.5(0-100)$ & \\
\hline & Physical role limitation & M1: $100.0(75-100)$ & M1: $100.0(50-100)$ & $\mathrm{p}=0.296$ \\
\hline & & IG time: $p=0.001^{\S}$ & CG time: $\mathrm{p}=0.001^{\S}$ & \\
\hline & & M0: $66.0(0-100)$ & M0: $66.0(0-100)$ & \\
\hline & Emotional role limitation & M1: $100.0(60-100)$ & M1: $100.0(33-100)$ & $\mathrm{p}=0.576$ \\
\hline & & IG time: $\mathrm{p}=0.001^{\S}$ & $C G^{\star}$ time: $\mathrm{p}=0.001^{\S}$ & \\
\hline & & M0: $54.73 \pm 13.69$ & M0: $54.70 \pm 13.26$ & \\
\hline & Energy & M1: $68.68 \pm 15.44$ & M1: $67.75 \pm 14.09$ & $\mathrm{p}=0.001^{\S}$ \\
\hline & & IG time: $p=0.001^{\S}$ & CG ${ }^{\star}$ time: $\mathrm{p}=0.001^{\S}$ & \\
\hline \multirow[t]{19}{*}{ Durmus et al. (2013) } & Subscale & US + EP (IG, N = 20) & $\mathrm{EP}(\mathrm{CG}, \mathrm{N}=20)$ & Significance IG vs CG \\
\hline & & M0: $70.00 \pm 13.3$ & M0: $73.25 \pm 12.6$ & \\
\hline & Physical function & M1: $87.00 \pm 11.4$ & M1: $89.75 \pm 11.1$ & $\mathrm{p}=0.021^{\S}$ \\
\hline & & IG ${ }^{\star}$ time: $p=0.001^{\S}$ & $\mathrm{CG}^{\star}$ time: $\mathrm{p}=0.001^{\S}$ & \\
\hline & & M0: $58.40 \pm 10.8$ & M0: $56.60 \pm 10.1$ & \\
\hline & Mental health & $\mathrm{M} 1: 73.40 \pm 12.2$ & M1: $74.10 \pm 10.1$ & $\mathrm{p}=0.063$ \\
\hline & & IG time: $p=0.001^{\S}$ & CG time: $\mathrm{p}=0.001^{\S}$ & \\
\hline & & M0: $58.65 \pm 16.23$ & M0: $65.50 \pm 17.69$ & \\
\hline & Pain & M1: $77.20 \pm 11.44$ & M1: $77.45 \pm 12.48$ & $\mathrm{p}=0.046^{\S}$ \\
\hline & & $\mathrm{IG}^{\star}$ time: $\mathrm{p}=0.001^{\S}$ & $\mathrm{CG}^{\star}$ time: $\mathrm{p}=0.001^{\S}$ & \\
\hline & & M0: $51.75 \pm 15.24$ & M0: $50.25 \pm 14.46$ & \\
\hline & General health & M1: $61.00 \pm 16.59$ & M1: $66.75 \pm 14.26$ & $\mathrm{p}=0.180$ \\
\hline & & IG time: $p=0.001^{\S}$ & CG time: $\mathrm{p}=0.001^{\S}$ & \\
\hline & & M0: $54.45 \pm 13.57$ & M0: $58.85 \pm 14.39$ & \\
\hline & Social function & M1: $84.35 \pm 12.01$ & M1: $86.10 \pm 13.09$ & $\mathrm{p}=0.246$ \\
\hline & & IG ${ }^{*}$ time: $p=0.001^{\S}$ & $C G^{\star}$ time: $\mathrm{p}=0.001^{\S}$ & \\
\hline & & M0: $56.25 \pm 33.3$ & M0: $60.65 \pm 31.2$ & \\
\hline & Physical role limitation & M1: $96.75 \pm 8.1$ & M1: $90.75 \pm 15.2$ & $\mathrm{p}=0.285$ \\
\hline & & IG ${ }^{\star}$ time: $p=0.001^{\S}$ & $\mathrm{CG}^{\star}$ time: $\mathrm{p}=0.001^{\S}$ & \\
\hline
\end{tabular}




\section{Continued}

\begin{tabular}{|c|c|c|c|c|}
\hline & Emotional role limitation & 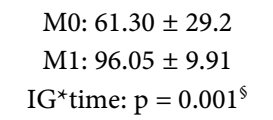 & 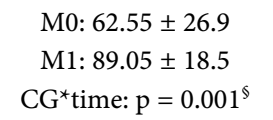 & $\mathrm{p}=0.258$ \\
\hline & Energy & $\begin{array}{c}\text { M0: } 55.75 \pm 14.0 \\
\text { M1: } 69.00 \pm 15.09 \\
\text { IG }^{*} \text { time: } p=0.001^{\S}\end{array}$ & 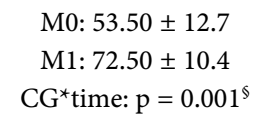 & $\mathrm{p}=0.009^{\varsigma}$ \\
\hline \multirow[t]{9}{*}{ Durmus et al. (2014) } & Subscale & $\begin{array}{c}M W D+E P \\
(I G, N=19)\end{array}$ & $\mathrm{EP}(\mathrm{CG}, \mathrm{N}=20)$ & Significance IG vs CG \\
\hline & Physical function & $\begin{array}{c}\text { M0: } 76.84 \pm 11.92 \\
\text { M1-M0: } 14.47 \pm 9.26 \\
\text { M2-M0: } 12.89 \pm 9.90\end{array}$ & $\begin{array}{c}\text { M0: } 74.50 \pm 12.01 \\
\text { M1-M0: } 16.25 \pm 8.12 \\
\text { M2-M0: } 14.25 \pm 8.19\end{array}$ & $\begin{array}{l}p=0.396 \\
p=0.771 \\
p=0.901\end{array}$ \\
\hline & Social function & $\begin{array}{c}\text { M0: } 53.94 \pm 16.20 \\
\text { M1-M0: } 11.10 \pm 10.80 \\
\text { M2-M0: } 10.05 \pm 9.58\end{array}$ & $\begin{array}{c}\text { M0: } 67.70 \pm 18.32 \\
\text { M1-M0: } 11.85 \pm 11.30 \\
\text { M2-M0: } 9.00 \pm 9.15\end{array}$ & $\begin{array}{l}p=0.835 \\
p=0.771 \\
p=0.749\end{array}$ \\
\hline & Physical role limitation & $\begin{array}{c}\text { M0: } 67.78 \pm 28.06 \\
\text { M1-M0: } 26.25 \pm 24.9 \\
\text { M2-M0: } 0.56 \pm 0.47\end{array}$ & $\begin{array}{c}\text { M0: } 59.40 \pm 31.14 \\
\text { M1-M0: } 31.10 \pm 27.10 \\
\text { M2-M0: } 0.43 \pm 0.57\end{array}$ & $\begin{array}{l}\mathrm{p}=0.444 \\
\mathrm{p}=0.396 \\
\mathrm{p}=0.380\end{array}$ \\
\hline & Emotional role limitation & $\begin{array}{c}\text { M0: } 66.84 \pm 25.99 \\
\text { M1-M0: } 23.31 \pm 21.6 \\
\text { M2-M0: } 17.78 \pm 18.9\end{array}$ & $\begin{array}{c}\text { M0: } 64.20 \pm 25.99 \\
\text { M1-M0: } 27.30 \pm 23.4 \\
\text { M2-M0: } 24.55 \pm 21.6\end{array}$ & $\begin{array}{l}p=0.771 \\
p=0.380 \\
p=0.336\end{array}$ \\
\hline & Mental health & $\begin{array}{c}\text { M0: } 62.84 \pm 13.54 \\
\text { M1-M0: } 14.73 \pm 10.38 \\
\text { M2-M0: } 12.94 \pm 9.47\end{array}$ & $\begin{array}{c}\text { M0: } 60.20 \pm 12.68 \\
\text { M1-M0: } 15.95 \pm 10.86 \\
\text { M2-M0: } 13.75 \pm 8.79\end{array}$ & $\begin{array}{l}\mathrm{p}=0.588 \\
\mathrm{p}=0.813 \\
\mathrm{p}=0.687\end{array}$ \\
\hline & Energy & $\begin{array}{c}\text { M0: } 62.36 \pm 8.22 \\
\text { M1-M0: } 17.63 \pm 10.05 \\
\text { M2-M0: } 13.94 \pm 8.75\end{array}$ & $\begin{array}{c}\text { M0: } 57.00 \pm 11.85 \\
\text { M1-M0: } 17.50 \pm 14.64 \\
\text { M2-M0: } 14.75 \pm 14.18\end{array}$ & $\begin{array}{l}p=0.127 \\
p=0.708 \\
p=0.771\end{array}$ \\
\hline & Pain & $\begin{array}{c}\text { M0: } 61.94 \pm 12.81 \\
\text { M1-M0: } 25.47 \pm 13.50 \\
\text { M2-M0: } 21.73 \pm 13.53\end{array}$ & $\begin{array}{c}\text { M0: } 57.75 \pm 13.76 \\
\text { M1-M0: } 28.60 \pm 12.54 \\
\text { M2-M0: } 24.65 \pm 12.99\end{array}$ & $\begin{array}{l}p=0.336 \\
p=0.749 \\
p=0.667\end{array}$ \\
\hline & General health & $\begin{array}{c}\text { M0: } 53.94 \pm 16.20 \\
\text { M1-M0: } 16.57 \pm 14.62 \\
\text { M2-M0: } 13.68 \pm 13.10\end{array}$ & $\begin{array}{c}\text { M0: } 52.00 \pm 14.72 \\
\text { M1-M0: } 15.75 \pm 12.06 \\
\text { M2-M0: } 13.00 \pm 9.65\end{array}$ & $\begin{array}{l}\mathrm{p}=0.569 \\
\mathrm{p}=0.989 \\
\mathrm{p}=0.901\end{array}$ \\
\hline \multirow[t]{2}{*}{ Licciardone et al. (2013) } & Total Score & US(IG, $N=233$ ) & $s U S(C G, N=222)$ & Significance IG vs CG \\
\hline & SF-36 & $\begin{array}{l}\text { M0: } 72(56-85) \\
\text { M1: } 72(54-87) \\
\text { M2: } 72(54-85) \\
\text { M3: } 72(52-87)\end{array}$ & $\begin{array}{l}\text { M0: } 67(52-82) \\
\text { M1: } 72(52-82) \\
\text { M2: } 72(57-85) \\
\text { M3: } 74(54-87)\end{array}$ & $\begin{array}{l}\text { M1: } p=0.73 \\
\text { M2: } p=0.53 \\
\text { M3: } p=0.66\end{array}$ \\
\hline
\end{tabular}

Legend. M0, M1, M2, M3 = measurement time points, see Figure 4 for clarification, IG = intervention group, CG = control group, US = ultrasound, EP = exercise program, $\mathrm{sUS}=$ sham ultrasound, $\mathrm{MWD}=$ microwave diathermy. ${ }^{5}=$ significant at $\alpha<0.05$. 